\title{
PD-I/PD-LI Inhibitor Combined with Chemotherapy Can Improve the Survival of Non-Small Cell Lung Cancer Patients with Brain Metastases
}

This article was published in the following Dove Press journal: OncoTargets and Therapy

\author{
Chenglong Sun ${ }^{1,2, *}$ \\ Fei Zhou ${ }^{3, *}$ \\ Xuefei $\mathrm{Li}^{4}$ \\ Chao Zhao ${ }^{4}$ \\ Wei $\mathrm{Li}^{3}$ \\ Jiayu $\mathrm{Li}^{3}$ \\ Anwen Xiong ${ }^{3}$ \\ Jia $\mathrm{Yu}^{3}$ \\ Guanghui $\mathrm{Gao}^{3}$ \\ Qi Wang (iD ${ }^{3}$ \\ Fengying $\mathrm{Wu}^{3}$ \\ Caicun Zhou ${ }^{3}$
}

\begin{abstract}
'Radiotherapy Department, Anhui No. 2 Provincial People's Hospital, Hefei 23004I, Anhui, People's Republic of China; ${ }^{2}$ Medical College of Soochow University, Suzhou 215I23, Jiangsu, People's Republic of China; ${ }^{3}$ Department of Medical Oncology, Shanghai Pulmonary Hospital, Tongji University Medical School Cancer Institute, Tongji University School of Medicine, Shanghai 200433, People's Republic of China; ${ }^{4}$ Department of Lung Cancer and Immunology, Shanghai Pulmonary Hospital \& Thoracic Cancer Institute, Tongji University School of Medicine, Shanghai 200433,

People's Republic of China

*These authors contributed equally to this work
\end{abstract}

Correspondence: Fengying Wu; Caicun

Zhou

Department of Medical Oncology, Shanghai Pulmonary Hospital, Tongji University Medical School Cancer Institute, Tongji University School of Medicine, No. 507

Zhengmin Road, Shanghai 200433, People's Republic of China

Tel +086 I3 | 67060870; +086 | 330 | 825532

Email fywu@163.com;

caicunzhoudr@I26.com
Introduction: Immune checkpoint inhibitor (ICI) monotherapy has limited efficacy in patients with non-small cell lung cancer (NSCLC) and brain metastases (BMs). With the wide use of ICI-based combinations, the efficacy of different ICI combination strategies in patients with NSCLC and BMs needs to be further elucidated.

Methods: We retrospectively reviewed 526 patients with non-small cell lung cancer (NSCLC) treated with ICIs from January 2016 to December 2019 in the Shanghai Pulmonary Hospital. Patients with BMs treated with ICIs were further divided into two groups: those with BM prior to the ICI treatment (pBM group), and those with BM after the treatment (aBM group). We assessed intracranial progression-free survival (IPFS), systemic progression-free survival (SPFS), overall survival (OS), intracranial objective response rate (IORR), and intracranial disease control rate (IDCR).

Results: We found 77 patients out of 526 with BMs; 69 presented the BMs prior to the ICI treatments and 8 showed BMs after the ICI treatments. In the pBM group, the median IPFS and SPFS were 7.39 months and 5.39 months, respectively. Combination therapy significantly improved both the IPFS $(p=0.007)$ and the SPFS $(p=0.007)$ when compared with monotherapy. Further analysis demonstrated that ICIs combined with chemotherapy significantly improved both the IPFS $(p=0.009)$ and the SPFS $(p=0.006)$ when compared with monotherapy. While ICIs combined with anti-angiogenic therapy improved the SPFS ( $p=0.005$ ) but not the IPFS ( $p=0.139)$. The median OS was 27.43 months for patients in the pBM group. Further analyses suggested that combination treatment also improved the OS when compared with monotherapy $(p=0.003)$. Subgroup analysis results showed that ICIs combined with chemotherapy led to better OS than ICIs monotherapy $(p=0.006)$. Radiotherapy had no significant impact on survival (IPFS $p=0.272$, OS $p=0.142$ ) in the patients of the pBM group.

Conclusion: ICIs combined with chemotherapy demonstrated survival benefits over ICI monotherapy in patients with NSCLCs and BMs.

Keywords: NSCLC, immune checkpoint inhibitors, brain metastases, survival, chemotherapy, anti-angiogenesis

\section{Introduction}

The brain is a common metastatic site of NSCLCs; approximately $10 \%$ of patients present BMs at the time of diagnosis and approximately $30 \%$ of BMs develop subsequently. ${ }^{1,2}$ Patients with BMs have poor survivals, with a median survival of 7 months. ${ }^{3}$ Several local treatments including metastasectomy and radiotherapy have been widely applied to improve local control, but they decrease cognitive ability 
and quality of life. ${ }^{4-7}$ Thus, systemic treatments are recommended for patients with asymptomatic or minimal neurological symptoms because these treatments provide disease control for extracranial tumors at the same time. ${ }^{8}$ Inhibitors targeting PD-1 or PD-L1 have achieved great improvements in overall survival (OS) for advanced NSCLC. ${ }^{9-11}$ However, patients with BM have been excluded from several clinical trials on inhibitors targeting PD-1 or PD-L1 due to their uncertain efficacy for metastatic brain lesions. ${ }^{12-14}$ Recent data have confirmed that immunotherapy is active against metastatic intracranial lesions in cases with NSCLC or melanoma. Furthermore, immune checkpoint inhibitors (ICIs) have been shown to inhibit the incidence of BMs in patients with NSCLC. ${ }^{15,16}$ Several treatment strategies including ICI monotherapy, combination with chemotherapy, and combination with anti-angiogenesis agents have been approved or are being investigated in NSCLCs. But, the most effective strategy for patients with BMs remains unknown. A previous study indicated that the combination of nivolumab with ipilimumab had a better response for intracranial lesions than either ipilimumab or pembrolizumab alone in patients with melanoma and BMs. ${ }^{17-19}$ Radiotherapy is very important for BMs. ICIs and concurrent stereotactic radiosurgery. (SRS can improve intracranial control). ${ }^{20-23}$ Meanwhile, another study showed that radiotherapy combined with ICIs does not provide survival benefits for NSCLC. ${ }^{24}$ Whether the efficacy of different strategies on intracranial and extracranial lesions is consistent is uncertain, and the question remains: Which strategy is optimal for BMs in patients with NSCLC?

Thus, we retrospectively analyzed treatments and outcomes of patients with NSCLC and BMs who received anti-PD-1/PD-L1-based treatments in the Shanghai Pulmonary Hospital, to find optimal anti-PD-1/PD-L1based treatment strategies for this specific patients' subgroup.

\section{Patients and Methods}

We conducted a retrospective study on patients with NSCLC and BMs who received anti-PD-1/PD-L1-based treatment in the Shanghai Pulmonary Hospital from January 2016 to December 2019. BMs were confirmed by either brain magnetic resonance imaging (MRI) or enhanced brain computed-tomography (CT). Anti-PD-1/ PD-L1-based treatments were used as single agents or in combination with chemotherapy or anti-angiogenesis agents. Intracranial and extracranial tumor responses were evaluated using the Response Evaluation Criteria in Solid Tumors version 1.1 (RECIST 1.1) guidelines. We collected all detailed information about patients from electronic medical records. All patients signed written informed consents. The ethics committee of Shanghai Pulmonary Hospital approved this study. The study was performed in accordance with the International Conference on Harmonisation Guidelines on Good Clinical Practice and the Declaration of Helsinki.

\section{Statistical Analysis}

We defined the intracranial disease control rate (IDCR) as the proportion of cases with complete, partial response, or stable disease. The intracranial objective response rate (IORR) was the proportion of patients with complete or partial response. OS was calculated from the date of diagnosis until death due to any cause. Systemic immunotherapy progression-free survival (SPFS) was calculated from the date of the first immunotherapy administration until progressive disease (PD) or death due to any cause. Intracranial immunotherapy progression-free survival (IPFS) was calculated from the date of the first immunotherapy administration until BM progressive disease (PD) or death due to any cause. We applied a Cox proportional hazards regression model to evaluate factors independently associated with OS and IPFS. We selected the variables included in the final multivariate model according to their clinical relevance and statistical significance in a univariate analysis (cutoff $\mathrm{p}=0.05$ ). We performed statistical analyses using SPSS 25.0 (IBM) and GraphPad Prism version 8.0 (GraphPad Software) for Mac OS.

\section{Results}

\section{Patient Baseline Characteristics}

In all, 526 patients with NSCLC received ICIs during the study period; 77 presented BMs (69 diagnosed before the ICI treatment [pBM group] and 8 diagnosed after it [aBM group]) (Figure 1). As shown in Table 1, the median age was 59.6 years (range, 33-88 years), 57 patients (74.0\%) were men, $46(59.7 \%)$ were never smokers, in the pBM group 53 patients $(76.8 \%)$ had an ECOG performance status (PS) between 0 and 1, 16 had a PS of 2, and, in the aBM group 7 patients $(87.5 \%)$ had a PS of 2,1 $(12.5 \%)$ had a PS of 0 . In the pBM group, 47 patients had adenocarcinoma, 8 squamous carcinoma, and 14 other types of lung cancer ( 8 had NSCLCs, and 6 large cell carcinoma). In the aBM group, 6 patients had 


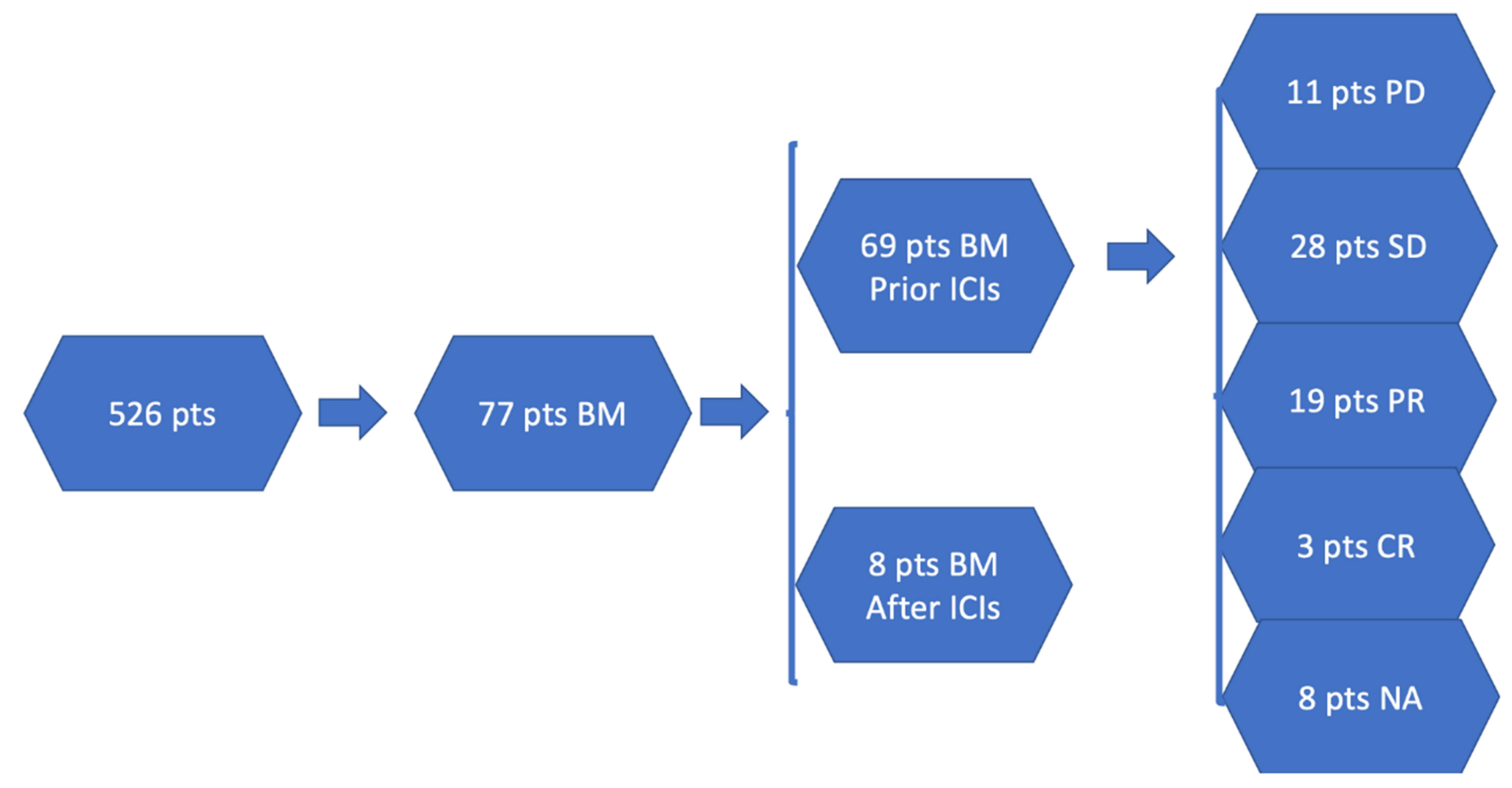

Figure I Diagram of the study.

Abbreviations: BMs, brain metastases; pts, patients; ICl, immune checkpoint inhibitors; CR, complete response; PR, partial response; SD, stable disease; PD, progressive disease; NA, not assessed.

adenocarcinoma and 2 squamous carcinoma. We found EGFR mutations $(14 / 77,18.2 \%)$, ALK rearrangements $(2 / 77,2.6 \%)$, KARS mutations $(6 / 77,7.8 \%)$, and ROS1 rearrangements $(1 / 77,1.3 \%)$ in the $\mathrm{pBM}$ group and 2 EGFR mutations in the aBM group. In the $\mathrm{pBM}$ group, we found 4 patients (5.8\%) with PD-L1 expression $\geq 50 \%$, $5(7.2 \%)$ with PD-L1 expression between 1 and 50\%, 14 (20.3\%) with PD-L1 expression $\leq 1 \%$, and 46 (66.7\%) with unknown PD-L1 status; in the aBM group, 4 patients (50\%) had negative PD-L1 expression, and 4 (50\%) had an unknown PD-L1 expression status. In addition, in the pBM group 19 patients (27.5\%) received ICIs as first-line treatment, $12(17.4 \%)$ as second-line treatment, and 38 $(55.1 \%)$ as third-line or later treatment; and, in the aBM group, 2 patients $(25 \%)$ received ICIs as first-line treatment, $1(12.5 \%)$ as second-line treatment, and $5(62.5 \%)$ as third-line or later treatment. In all, 41 patients received brain radiotherapy and 28 did not. Among those who received intracranial radiotherapy, 29 received whole brain radio therapy (WBRT) and $12 \mathrm{SRT}$. In the pBM group, the treatments included single ICI agent regimen (30 patients, 43.5\%), ICI combined with chemotherapy (29 patients, 42\%) and ICI combined with anti-angiogenesis (10 patients, $14.5 \%$ ). In the aBM group, 4 patients $(50 \%)$ were treated with ICI monotherapy, 2 (25\%) with ICI combined with chemotherapy, and 2 (25\%) with ICI combined with an anti-angiogenesis agent.

\section{Baseline Characteristics for Intracranial Lesions}

We found 16 patients $(23.2 \%)$ in the pBM group and 3 $(27.5 \%)$ in the aBM group with maximum tumor diameters $\geq 3 \mathrm{~cm}$. Also, $53(77.8 \%)$ patients in the $\mathrm{pBM}$ group and $5(62.5 \%)$ in the aBM group had tumor sizes smaller than $3 \mathrm{~cm}$. We found 19 patients $(27.5 \%)$ in the pBM group and $3(27.5 \%)$ in the aBM group with separate edemas $\geq 3 \mathrm{~cm}$. Meningeal metastases occurred in 11 patients $(15.9 \%)$ of the pBM group, but not in the patients of the aBM group. We found 24 patients (34.8\%) in the pBM group and $2(25 \%)$ in the aBM group with DS-GPA (disease-specific Graded Prognostic Assessment) scores between 0 and 1, 42 patients $(60.9 \%)$ in the pBM group and $6(75 \%)$ in the aBM group with DS-GPA scores between 1.5 and 2.5 , and 3 patients $(4.3 \%)$ in the pBM group with DS-GPA scores between 3 and 4. Table 2 shows the detailed data.

We also assessed associations between clinical factors and IPFS or SPFS. We identified ECOG PS, ICI treatment line, and combination strategy as predictive factors for IPFS in the univariate analysis and ECOG PS as an 
Table I Patient Characteristics $(n=77)$

\begin{tabular}{|c|c|c|}
\hline & pBM Group & aBM Group \\
\hline Characteristic & Total $(n=69)$ & Total $(n=8)$ \\
\hline \multicolumn{3}{|l|}{ Gender, n (\%) } \\
\hline Male & 51 (73.9\%) & $6(75 \%)$ \\
\hline Female & $18(26.1 \%)$ & $2(15 \%)$ \\
\hline \multicolumn{3}{|l|}{ Age, median } \\
\hline$<70$ & 62 (89.9\%) & $5(62.5 \%)$ \\
\hline$\geqq 70$ & $7(10.1 \%)$ & 3 (37.5\%) \\
\hline \multicolumn{3}{|l|}{ Smoking status, n (\%) } \\
\hline Non-Smoker & 40 (58\%) & $6(75 \%)$ \\
\hline Smoker & $29(42 \%)$ & $2(15 \%)$ \\
\hline \multicolumn{3}{|l|}{ ECOG PS } \\
\hline $0-1$ & $53(76.8 \%)$ & I (I2.5\%) \\
\hline$\geq 2$ & $16(23.2 \%)$ & 7 (87.5\%) \\
\hline \multicolumn{3}{|l|}{ Tumor Histology } \\
\hline Adenocarcinoma & $47(68.1 \%)$ & $6(75 \%)$ \\
\hline Squamous cell carcinoma & $8(\mathrm{II} .6 \%)$ & $2(15 \%)$ \\
\hline Other & $14(20.3 \%)$ & 0 \\
\hline \multicolumn{3}{|l|}{ Mutation } \\
\hline No-mutation & $48(69.6 \%)$ & $6(75 \%)$ \\
\hline EGFR mutation & $12(17.4 \%)$ & $2(15 \%)$ \\
\hline KRAS mutation & $6(8.7 \%)$ & 0 \\
\hline ALK mutation & $2(2.9 \%)$ & 0 \\
\hline ROSI mutation & $\mathrm{I}(\mathrm{I} .4 \%)$ & 0 \\
\hline \multicolumn{3}{|l|}{ PD-LI expression } \\
\hline NO-detected & $46(66.7 \%)$ & $4(50 \%)$ \\
\hline 0 & $14(20.3 \%)$ & $4(50 \%)$ \\
\hline $\mathrm{I}-50 \%$ & $5(7.2 \%)$ & \\
\hline$\geq 50 \%$ & $4(5.8 \%)$ & \\
\hline \multicolumn{3}{|l|}{$\begin{array}{l}\text { Number of prior systemic therapy } \\
\text { regimens before ICls }\end{array}$} \\
\hline 1 & $19(27.5 \%)$ & $2(25 \%)$ \\
\hline 2 & $12(17.4 \%)$ & I (12.5\%) \\
\hline$\geq 3$ & $38(55.1 \%)$ & $5(62.5 \%)$ \\
\hline \multicolumn{3}{|l|}{ Liver metastases } \\
\hline Yes & $3(4.3 \%)$ & I (I2.5\%) \\
\hline No & $66(95.7 \%)$ & 7 (87.5\%) \\
\hline \multicolumn{3}{|l|}{ Radiotherapy } \\
\hline NO-Radiotherapy & $28(40.6 \%)$ & 0 \\
\hline WBRT & $29(42 \%)$ & $3(37.5 \%)$ \\
\hline SRS & $12(17.4 \%)$ & $5(62.5 \%)$ \\
\hline \multicolumn{3}{|l|}{$\mathrm{ICls}$ regimes } \\
\hline Monotherapy & $30(43.5 \%)$ & $4(50 \%)$ \\
\hline Chemotherapy & $29(42 \%)$ & $2(25 \%)$ \\
\hline Anti-angiogenic therapy & $10(14.5 \%)$ & $2(25 \%)$ \\
\hline
\end{tabular}

Abbreviations: $\mathrm{ICl}$, immune checkpoint inhibitor; PD-LI, programmed deathligand I; WBRT, whole brain radiotherapy; SRS, stereotactic radiosurgery; PBM, patients with brain metastasis prior to ICls treatment; aBM group, patients with brain metastasis after ICls treatment.
Table 2 Characteristic of Intracranial Lesion at Start of ICls

\begin{tabular}{|c|c|c|c|}
\hline & pBM Group & $\mathrm{aBM} \mathbf{G r}$ & $\mathrm{p}$ value \\
\hline $\begin{array}{l}\text { Size of intracranial lesion } \\
\text { (maximum diameter) } \\
\quad \geq 3 \mathrm{~cm} \\
<3 \mathrm{~cm}\end{array}$ & $\begin{array}{l}16(23.2 \%) \\
53(77.8 \%)\end{array}$ & $\begin{array}{l}3(27.5 \%) \\
5(62.5 \%)\end{array}$ & $0.649 \&$ \\
\hline $\begin{array}{l}\text { Edema size } \\
\qquad \begin{array}{l}\geq 3 \mathrm{~cm} \\
<3 \mathrm{~cm}\end{array}\end{array}$ & $\begin{array}{l}19(27.5 \%) \\
50(72.5)\end{array}$ & $\begin{array}{l}3(27.5 \%) \\
5(62.5 \%)\end{array}$ & $0.895 \&$ \\
\hline $\begin{array}{l}\text { Number of total lesions } \\
\quad<3 \\
\geq 3\end{array}$ & $\begin{array}{l}35(50.7 \%) \\
34(49.3 \%)\end{array}$ & $\begin{array}{l}6(75 \%) \\
2(25 \%)\end{array}$ & $0.27 \mathrm{I} \#$ \\
\hline $\begin{array}{l}\text { Meningeal metastasis } \\
\text { Yes } \\
\text { No }\end{array}$ & $\begin{array}{l}\mathrm{II}(15.9 \%) \\
58(84.1 \%)\end{array}$ & $\begin{array}{l}0(0 \%) \\
8(100 \%)\end{array}$ & $0.493 \&$ \\
\hline $\begin{array}{l}\text { DS-GPA score } \\
0-1 \\
1.5-2.5 \\
3-4\end{array}$ & $\begin{array}{l}24(34.8 \%) \\
42(60.9 \%) \\
3(4.3 \%)\end{array}$ & $\begin{array}{l}2(25 \%) \\
6(75 \%) \\
0(0 \%)\end{array}$ & $0.677^{*}$ \\
\hline
\end{tabular}

Notes: *Pearson's chi-squared test; \# Fisher's Exact Test; \& Continuous correction. Abbreviations: ICls, immune checkpoint inhibitors; DS-GPA, disease-specific graded prognostic assessment; $\mathrm{pBM}$, patients with brain metastasis prior to $\mathrm{ICls}$ treatment; aBM group, patients with brain metastasis after ICls treatment.

independent predictive factor in the multivariate analysis. In addition, ECOG PS and the ICI combination strategy were independent predictive factors for SPFS in both the univariate and multivariate analyses (Tables 3 and 4).

\section{Survival Analysis}

The median IPFS was 7.39 months (95\% CI, 5.70-9.09) for patients in the $\mathrm{pBM}$ group. In the pBM group, we compared the survival in patients with ICI monotherapy with that in patients with combination treatments and found better survivals for patients who underwent combination treatment $(\mathrm{p}=0.007)$. Further subgroup analyses showed that patients undergoing ICI combined with chemotherapy had better IPFS than those undergoing ICI monotherapy $(\mathrm{p}=0.009)$. ICI combined with antiangiogenic therapy resulted in similar IPFS to the ICI monotherapy $(\mathrm{p}=0.139)$. The median SPFS was 5.39 months (95\% CI, 4.15-6.63) for patients in the pBM group. Combination therapy produced better survivals than monotherapy $(\mathrm{p}=0.007)$, and we found similar SPFSs in patients receiving either ICIs combined with chemotherapy or ICIs combined anti-angiogenesis agents $(\mathrm{P}=0.652)$ (Figures 2 and 3). 
Table 3 Cox Analysis of BM IPFS

\begin{tabular}{|c|c|c|c|c|c|c|c|c|}
\hline & \multicolumn{8}{|c|}{ BM IPFS (pBM Group) } \\
\hline & \multicolumn{4}{|c|}{ Univariate Analysis } & \multicolumn{4}{|c|}{ Multivariate Analysis } \\
\hline & \multirow[t]{2}{*}{ HR } & \multicolumn{2}{|c|}{$95 \% \mathrm{Cl}$} & \multirow[t]{2}{*}{ p value } & \multirow[t]{2}{*}{ HR } & \multicolumn{2}{|l|}{$95 \% \mathrm{Cl}$} & \multirow[t]{2}{*}{ p value } \\
\hline & & Down & Up & & & Down & Up & \\
\hline Age $(\geq 70$ vs $<70)$ & 1.67 & 0.496 & 5.618 & 0.408 & & & & \\
\hline \multicolumn{9}{|l|}{ Histologic type } \\
\hline Adeno vs Squamous & 1.753 & 0.404 & 7.602 & 0.453 & & & & \\
\hline Adeno vs Other & 0.989 & 0.638 & 1.535 & 0.962 & & & & \\
\hline ICls line, $\geq 3$ vs $<3$ & 2.225 & 1.071 & 4.621 & $0.032 *$ & $\mathrm{I} .44 \mathrm{I}$ & 0.624 & 3.332 & 0.393 \\
\hline ECOG PS, 2 vs $0-I$ & 4.485 & 2.153 & 9.346 & $<0.00 I^{*}$ & 3.247 & 1.448 & 7.283 & $0.004 *$ \\
\hline Size of intracranial lesion (maximum diameter) $(\geq 3 \mathrm{~cm}$ vs $<3 \mathrm{~cm}$ ) & 0.451 & 0.159 & 1.282 & 0.135 & & & & \\
\hline Edema size $(\geq 3 \mathrm{~cm}$ vs $<3 \mathrm{~cm})$ & 0.474 & 0.183 & 1.227 & 0.124 & & & & \\
\hline Number of total lesions & 1.2 & 0.621 & 2.32 & 0.588 & & & & \\
\hline Meningeal metastasis & 2.13 & 0.95 & 4.776 & 0.066 & & & & \\
\hline \multicolumn{9}{|l|}{ GPA score } \\
\hline $0-$ I vs I.5-2.5 & 0.924 & 0.396 & 2.157 & 0.856 & & & & \\
\hline $0-1$ vs 3 & 0.036 & 0 & 15,824 & 0.615 & & & & \\
\hline Combined with radiotherapy (yes vs no) & 0.697 & 0.36 & 1.346 & 0.282 & & & & \\
\hline ICls regimes (monotherapy vs combined therapy) & 0.386 & 0.196 & 0.759 & $0.006 *$ & 0.629 & 0.277 & 1.425 & 0.266 \\
\hline Mutation (EGFR mutation vs other mutation) & 1.051 & 0.643 & 1.718 & 0.842 & & & & \\
\hline WBRT (yes vs no) & 0.794 & 0.399 & 1.582 & 0.512 & & & & \\
\hline SRS (yes vs no) & 0.794 & 0.328 & 1.919 & 0.608 & & & & \\
\hline Liver metastasis & 1.328 & 0.316 & 5.580 & 0.699 & & & & \\
\hline Gender, n (\%) (male vs female) & 0.957 & 0.449 & 2.04 & 0.910 & & & & \\
\hline
\end{tabular}

Abbreviations: ICls, immune checkpoint inhibitors; DS-GPA, disease-specific graded prognostic assessment; ECOG PS, Eastern Cooperative Oncology Group performance status; EGFR, epidermal growth factor receptor; WBRT, whole brain radiotherapy; SRS, stereotactic radiosurgery; pBM, patients with brain metastasis prior to ICls treatment. ${ }^{*} \mathrm{p}<0.05$.

The intracranial response to immunotherapy was also analyzed. In the pBM group, the IORR was $36.07 \%$ (22/ 61 ) and the IDCR $81.97 \%(50 / 61)$; and, in the aBM group, the IORR was $50 \%(3 / 6)$ and the IDCR $66.7 \%(4 / 6)$ (Figure 4). We found 48 patients $(48 / 77,62.3 \%)$ who received radiation (WBRT or SRT), 31 (31/48, 64.58\%) prior to ICI treatment and $17(17 / 48,35.42 \%)$ after ICI treatment in both groups. We could not assess correlations between the PD-L1 status and the intracranial response due to missing PD-L1 status data.

Our results indicate that radiotherapy has no significant impact on the survival of patients with BMs (IPFS, $\mathrm{p}=0.272$; OS, $\mathrm{p}=0.142$ ).

The median OS was 27.43 months (95\% CI, 22.8232.04) for patients in the pBM group. We observed no significant differences in the survivals of patients between the $\mathrm{pBM}$ and the aBM groups $(\mathrm{p}=0.431)$. We found that the patients undergoing combination treatments had better survivals than those undergoing monotherapy $(\mathrm{p}=0.003)$ in the $\mathrm{pBM}$ group. We found similar survivals for patients in both combination regimens $(p=0.440)$. We could not assess survival in the aBM group due to the small sample size of the cohort.

\section{Discussion}

In this study, we analyzed the response to ICIs of patients with NSCLC and BMs and found that ICI combination treatments result in better IPFS and SPFS than ICI monotherapy. Further analyses suggested that this improvement was mainly due to combinations with chemotherapy $(p=0.009)$ and not with anti-angiogenesis agents $(p=0.139)$. Thus, our results indicate that ICI combination therapy may be superior for metastatic brain lesions than other treatment strategies, but we cannot conclude that ICI chemotherapy combinations are better than ICI anti-angiogenesis combinations due to the small sample size in our anti-angiogenesis cohort arm. However, in agreement with the findings in the report by Gauvain et $\mathrm{al}^{25}$ we confirmed that ICIs were 
Table 4 COX Analysis of SPFS

\begin{tabular}{|c|c|c|c|c|c|c|c|c|}
\hline & \multicolumn{8}{|c|}{ System-IPFS (pBM Group) } \\
\hline & \multicolumn{4}{|c|}{ Univariate Analysis } & \multicolumn{4}{|c|}{ Multivariate Analysis } \\
\hline & \multirow[t]{2}{*}{ HR } & \multicolumn{2}{|c|}{$95 \% \mathrm{Cl}$} & \multirow[t]{2}{*}{ p value } & \multirow[t]{2}{*}{ HR } & \multicolumn{2}{|l|}{$95 \% \mathrm{Cl}$} & \multirow[t]{2}{*}{ p value } \\
\hline & & Down & Up & & & Down & Up & \\
\hline Age ( $\geq 70$ vs $<70)$ & 1.248 & $0.44 I$ & 3.531 & 0.667 & & & & \\
\hline \multicolumn{9}{|l|}{ Histologic type } \\
\hline Adeno vs Squamous & 1.12 & 0.431 & 2.913 & 0.815 & & & & \\
\hline Adeno vs Other & 1.204 & 0.852 & 1.702 & 0.293 & & & & \\
\hline ICls line, $\geq 3$ vs $<3$ & 1.952 & 1.068 & 3.567 & 0.03 & & & & \\
\hline ECOG PS, 2 vs $0-1$ & 3.698 & 1.777 & 7.696 & $<0.001 *$ & 3.264 & 1.766 & 6.033 & $<0.001 *$ \\
\hline Size of intracranial lesion (maximum diameter) $(>3 \mathrm{~cm}$ vs $<3 \mathrm{~cm}$ ) & 1.096 & 0.557 & 2.159 & 0.791 & & & & \\
\hline Edema size $(>3 \mathrm{~cm}$ vs $<3 \mathrm{~cm})$ & 0.972 & 0.503 & 1.877 & 0.933 & & & & \\
\hline Number of total lesions & 1.27 & 0.718 & 2.246 & 0.412 & & & & \\
\hline Meningeal metastasis & 1.408 & 0.668 & 2.966 & 0.369 & & & & \\
\hline \multicolumn{9}{|l|}{ GPA score } \\
\hline $0-1$ vs $1.5-2.5$ & 0.995 & 0.54 & 1.834 & 0.987 & & & & \\
\hline $0-1$ vs 3 & 2.423 & 0.538 & 10.911 & 0.249 & & & & \\
\hline Combined with radiotherapy (yes vs no) & 0.862 & 0.484 & 1.536 & 0.615 & & & & \\
\hline ICls regimes (monotherapy vscombined therapy) & 0.368 & 0.206 & 0.657 & $0.001 *$ & 0.52 & 0.279 & 0.967 & $0.039 *$ \\
\hline Mutation (EGFR mutation vs other mutation) & 0.791 & 0.272 & 2.3 & 0.667 & & & & \\
\hline Liver metastasis & 1.210 & 0.372 & 3.934 & 0.751 & & & & \\
\hline Gender, n (\%) (male vs female) & 1.226 & 0.647 & 2.325 & 0.532 & & & & \\
\hline
\end{tabular}

Note: ${ }^{*} \mathrm{p}<0.05$.

Abbreviations: ICls, immune checkpoint inhibitors; DS-GPA, disease-specific graded prognostic assessment; ECOG PS, Eastern Cooperative Oncology Group performance status; EGFR, epidermal growth factor receptor; pBM, patients with brain metastasis prior to ICls treatment.

effective to treat intracranial lesions, with similar responses for intracranial and extracranial lesions.

Pembrolizumab and nivolumab as monotherapy showed efficacy against BMs in melanoma and nonsmall cell lung cancer (NSCLC). ${ }^{19,26,27}$ Combination therapies with ipilimumab have been common in patients with melanoma and BMs, and they result in meaningful intracranial efficacy. ${ }^{17,28}$ Nivolumab and ipilimumab had a CNS response rate of $46 \%$, while nivolumab alone had a CNS response rate of $20 \% .^{29}$ ICI combination chemotherapies have achieved positive extracranial efficacy in many clinical studies of non-small cell lung cancer, $^{12,30-32}$ but those studies have excluded patients with symptomatic or untreated BMs, and intracranial responses have not been reported in patients with NSCLCs. The effect of ICIs combined with chemotherapy on BMs is still uncertain. Our retrospective analysis showed that ICIs combined with chemotherapy produced a better intracranial response than that produced by ICIs alone.
In this study, the combination of ICIs and antiangiogenic therapy resulted in similar IPFS than the combination of ICIs and chemotherapy $(p=0.139)$. Due to the retrospective nature of our study and the small sample, larger studies are needed to further clarify differences between different combination therapies. We are interested in the results of two Phase II clinical trials that are presently recruiting patients, one on combination therapy with pembrolizumab and bevacizumab in patients with BMs and untreated NSCLC or melanoma (NCT02681549) and another one on combination therapy with atezolizumab and bevacizumab for patients with BMs and untreated melanoma (NCT03175432).

Our results showed no OS effects after local brain radiotherapy in the patients of the pBM group. The abscopal effect occurs when local tumor radiation causes regression of distant metastatic sites outside of the radiation field. ${ }^{33}$ ICIs have been shown to improve the abscopal effect of radiation in pre-clinical models. ${ }^{33,34}$ Previous studies have shown that concurrent stereotactic 

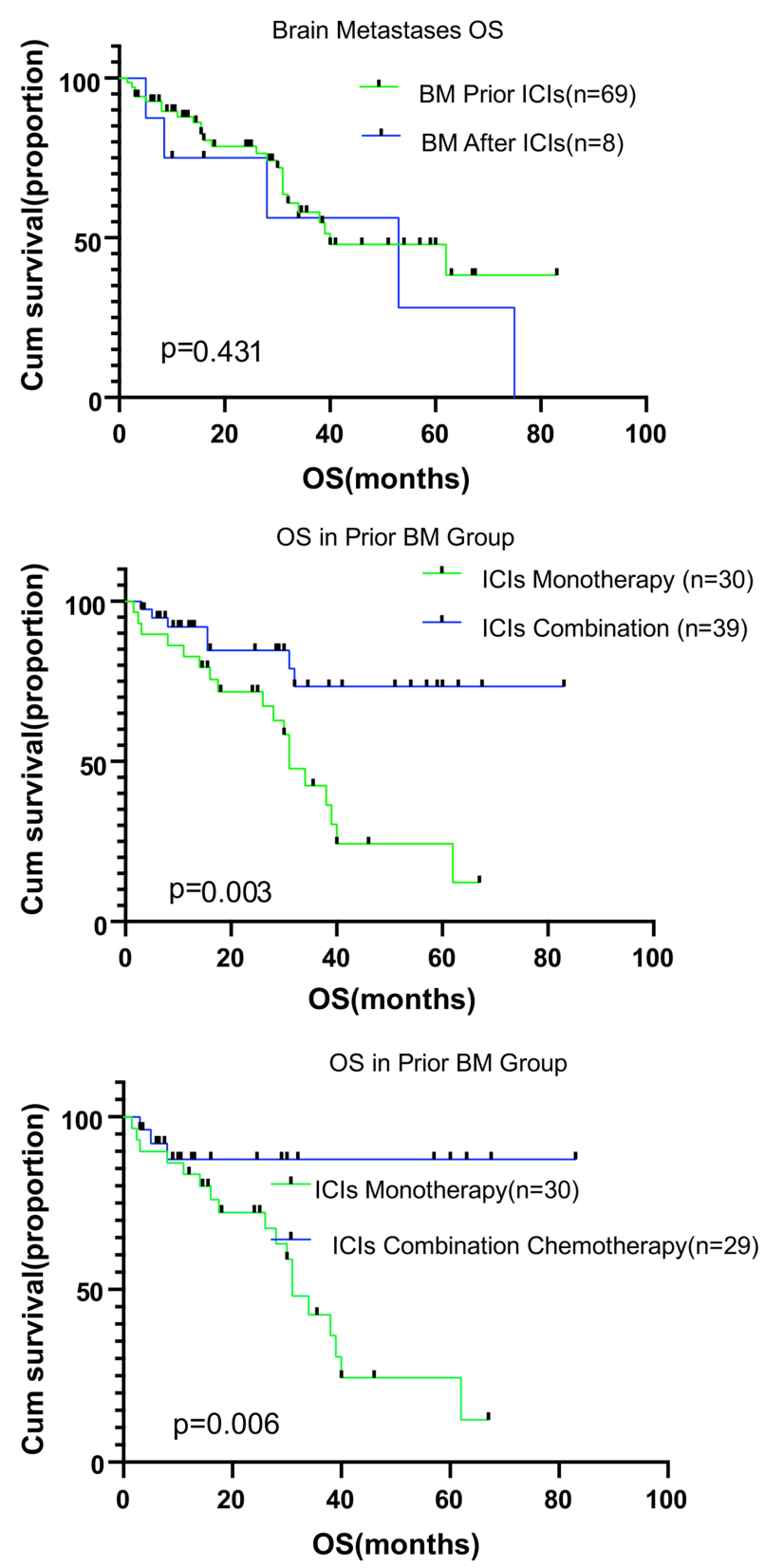
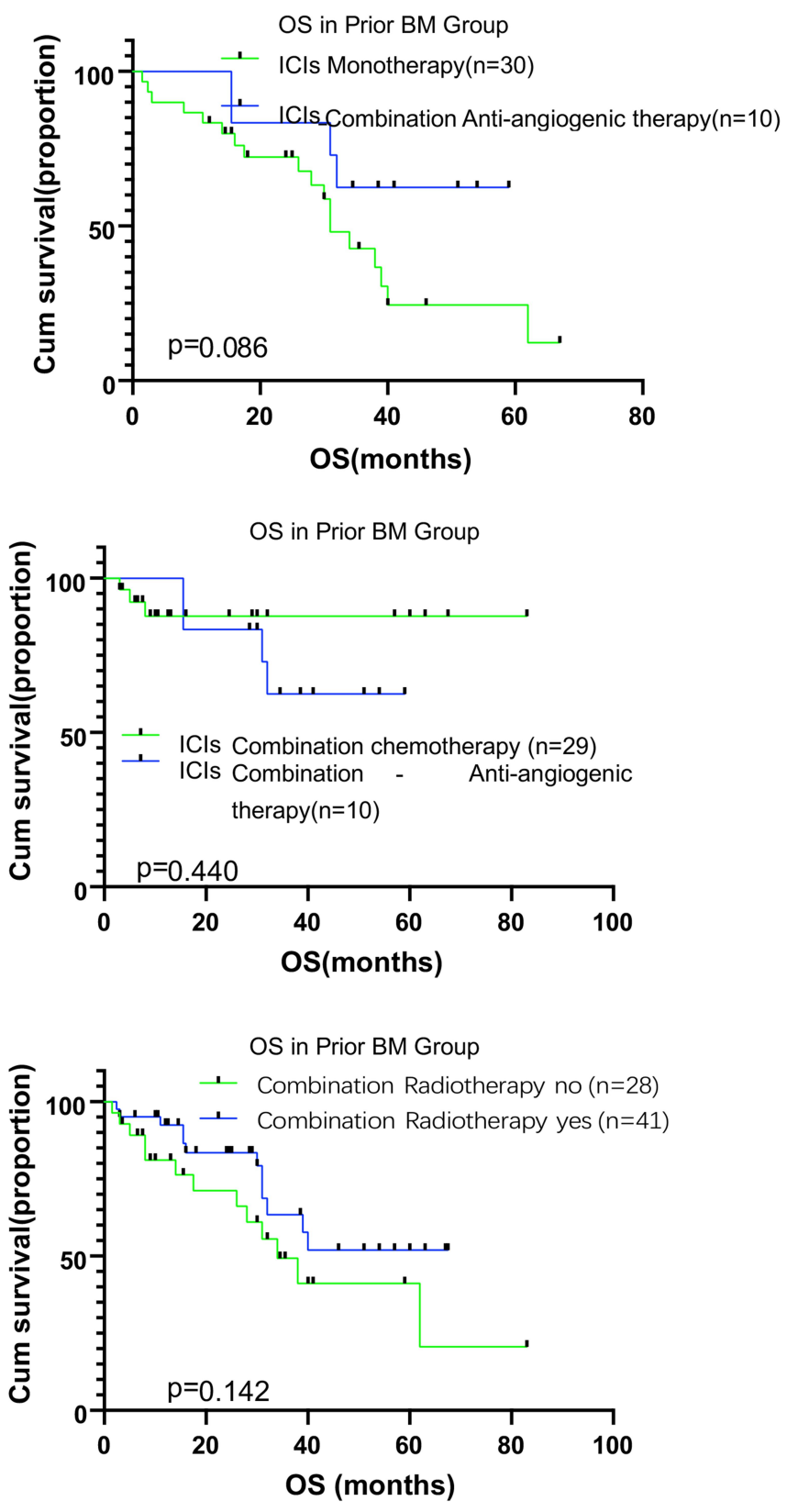

Figure 2 Kaplan-Meier analysis for overall survival in patients with brain metastases according to therapy type. $\mathrm{ICl}$ monotherapy, ICl combined therapy, ICl combined chemotherapy, ICl combined anti-angiogenic therapy, and radiotherapy for brain metastases. The respective log-rank $\mathrm{P}$ values are shown in the chart. Abbreviation: OS, overall survival.

radiosurgery (SRS)/SRT and ICI treatments produced an OS benefit compared to SRS/SRT alone. ${ }^{23,35}$ Moreover, concurrent administration of SRS and immunotherapy led to better outcomes in terms of response and survival for patients with BMs than non-concurrent SRS or ICI treatments. $^{23,36}$ Ahmed et al reported that SRS for BMs administered during or before ICI treatment led to better 6-month local control rates than SRS administered after ICI treatment in patients with NSCLC. ${ }^{37}$ Our results were consistent with those by Singh et al, showing that
SRT combined with ICIs produces no significant benefit in the survival or response rate of patients with BMs. ${ }^{24}$ Concurrent radiotherapy and immunotherapy were not administrated in our study cohort. The patients in our study received either WBRT or SRT (29 patients [42\%] received WBRT). Many studies have indicated that concurrent SRT and ICIs can lead to survival benefits in patients with BMs, but few studies have focused on WBRT combined with ICIs. Demaria et al reported that concurrent radiation with ICIs was more effective than 

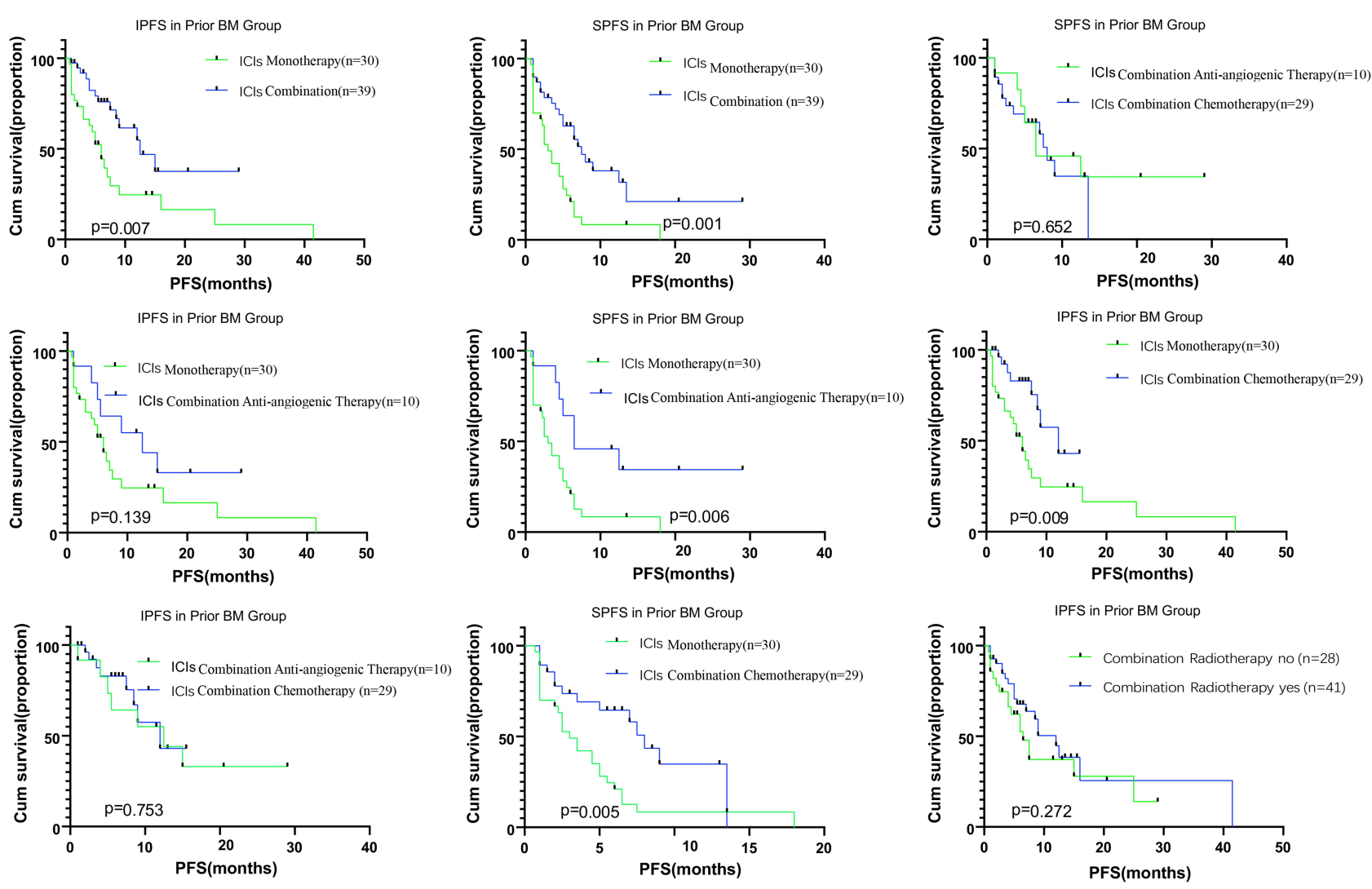

Figure 3 Kaplan-Meier analysis for systemic and brain metastases in intracranial progression-free survival according to therapy type. ICI monotherapy, ICl combined therapy, ICl combined chemotherapy, ICl combined anti-angiogenic therapy, and radiotherapy for brain metastases. The respective log-rank $\mathrm{P}$ values are shown in the chart. Abbreviations: IPFS, intracranial immunotherapy progression-free survival; SPFS, systemic immunotherapy progression-free survival; BM, brain metastases.

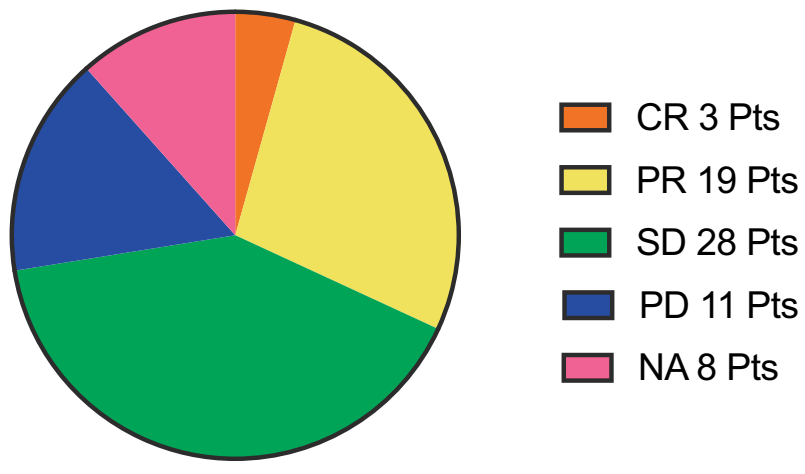

Total $=69$

Figure 4 Intracranial response in the $\mathrm{pBM}$ group. This pie chart shows 3 patients with CR, 19 with PR, 28 with SD, II with PD, and 8 NA (not assessed). The response of intracranial ORR was $31.9 \%$, IDCR $72.5 \%$ in prior BM group.

Abbreviations: BMs, brain metastases; pts, patients; ICls, immune checkpoint inhibitors; CR, complete response; PR, partial response; SD, stable disease; PD, progressive disease; NA, not assessed; ORR, objective response rate; IDCR, intracranial disease control rate.

sequential treatment and that high-dose hypofractionated radiation resulted in a better response. ${ }^{38}$ Concurrent WBRT with ICIs had no influence on the intracranial response in our study, but this may be due to the higher proportion of patients receiving WBRT than SRSand to the retrospective nature and relatively small sample size of our study. Whether radiation combined with ICI benefits patients with BMs needs further verification. ${ }^{22}$

Overall, in our study, combination therapy with ICIs led to a better OS than monotherapy $(\mathrm{p}=0.003)$; antiangiogenic therapy with ICIs and monotherapy led to similar survivals $(\mathrm{p}=0.086)$; and ICIs with chemotherapy resulted in a better OS than monotherapy $(\mathrm{p}=0.006)$.

In this study, we found an intracranial ORR of $36.07 \%$, which is consistent with reported ones at $27.3 \%,{ }^{39} 33 \%{ }^{19}$ and Goldberg et al reported a brain metastasis response of $29.7 \%$ on PD-L1 positive patients, ${ }^{40}$ we failed to analyze subgroup intracranial response and survival based on the expression of PD-L1 due to missing PD-L1 status data, this is also the limitation of this research.

ICIs combined with chemotherapy resulted in better survivals than monotherapy in many studies focused on extracranial lesions. ${ }^{30-32}$ However, these studies excluded 
symptomatic central nervous system metastases, so we found no data for comparison with ours.

Our results suggest that ICIs can achieve prompt control of both extracranial metastases and BMs in patients with NSCLC. Compared with monotherapy, ICIs combined with chemotherapy showed better survivals for patients with either extracranial or intracranial lesions in our study. Thus, combination of immunotherapy and chemotherapy may delay the need for radiotherapy, reduce the complications of WBRT and SRT (ie, cognitive decline and radiation necrosis, respectively), and improve the quality of life of patients with BM. Our results need to be confirmed in other studies.

\section{Conclusion}

ICIs can improve the survival of patients with NSCLC and BMs. ICIs were effective for NSCLC intracranial lesions, especially when combined with chemotherapy. ICIs showed consistent efficacy both for intracranial and extracranial lesions.

\section{Acknowledgments}

This study was supported in part by the Key Program of Natural Science Research of Anhui Provincial Education Department (KJ2018A0813), a grant from the National Natural Science Foundation of China (81802255). First authors: Chenglong Sun and Fei Zhou.

\section{Disclosure}

The authors report no conflicts of interest for this work.

\section{References}

1. Sorensen JB, Hansen HH, Hansen M, Dombernowsky P. Brain metastases in adenocarcinoma of the lung: frequency, risk groups, and prognosis. J Clin Oncol. 1988;6(9):1474-1480. doi:10.1200/JCO.19 88.6.9.1474

2. Gavrilovic IT, Posner JB. Brain metastases: epidemiology and pathophysiology. J Neurooncol. 2005;75(1):5-14. doi:10.1007/s1106 0-004-8093-6

3. Sperduto PW, Kased N, Roberge D, et al. Summary report on the graded prognostic assessment: an accurate and facile diagnosis-specific tool to estimate survival for patients with brain metastases. J Clin Oncol. 2012;30(4):419-425. doi:10.1200/JCO.20 11.38.0527

4. Mathieu D, Kondziolka D, Cooper PB, et al. Gamma knife radiosurgery for malignant melanoma brain metastases. Clin Neurosurg. 2007;54:241-247.

5. Khan AJ, Dicker AP. On the merits and limitations of whole-brain radiation therapy. J Clin Oncol. 2013;31(1):11-13. doi:10.1200/JCO. 2012.46.0410

6. Alomari A, Rauch PJ, Orsaria M, et al. Radiologic and histologic consequences of radiosurgery for brain tumors. $J$ Neurooncol. 2014;117(1):33-42. doi:10.1007/s11060-014-1359-8
7. Greene-Schloesser D, Robbins ME. Radiation induced cognitive impairment from bench to bedside. Neuro-Oncology. 2012;4 Suppl (4):iv37-iv44.

8. Peters S, Adjei AA, Gridelli C, et al. Metastatic non-small-cell lung cancer (NSCLC): ESMO clinical practice guidelines for diagnosis, treatment and follow-up. Ann Oncol. 2012;23(Suppl 7):vii56-vii64. doi:10.1093/annonc/mds226

9. Garon EB, Rizvi NA, Hui R, et al. Pembrolizumab for the treatment of non-small-cell lung cancer. $N$ Engl $J$ Med. 2015;372 (21):2018-2028. doi:10.1056/NEJMoa1501824

10. Brahmer J, Reckamp KL, Baas P, et al. Nivolumab versus docetaxel in advanced squamous-cell non-small-cell lung cancer. $N$ Engl $J$ Med. 2015;373(2):123-135. doi:10.1056/NEJMoa1504627

11. Borghaei H, Paz-Ares L, Horn L, et al. Nivolumab versus docetaxel in advanced nonsquamous non-small-cell lung cancer. $N$ Engl $J$ Med. 2015;373(17):1627-1639. doi:10.1056/NEJMoa1507643

12. Socinski MA, Jotte RM, Cappuzzo F, et al. Atezolizumab for first-line treatment of metastatic nonsquamous NSCLC. $N$ Engl J Med. 2018;378(24):2288-2301. doi:10.1056/NEJMoa1716948

13. Reck M, Rodríguez-Abreu D, Robinson AG. Pembrolizumab versus chemotherapy for PD-L1-positive non-small-cell lung cancer. $N$ Engl J Med. 2016;375(19):1823-1833. doi:10.1056/NEJMoa16 06774

14. Herbst RS, Baas P, Kim D-W, et al. Pembrolizumab versus docetaxel for previously treated, PD-L1-positive, advanced non-small-cell lung cancer (KEYNOTE-010): a randomised controlled trial. Lancet. 2016;387(10027):1540-1550. doi:10.1016/S0140-6736(15)01281-7

15. Ashinuma H, Shingyoji M, Iuchi T, et al. P2.07-014 immune checkpoint inhibitors for brain metastases of non-small-cell lung cancer. J Thorac Oncol. 2017;12(11):S2420. doi:10.1016/j.jtho.2017.11.073

16. Antonia SJ, Villegas A, Daniel D, et al. Durvalumab after chemoradiotherapy in stage III non-small-cell lung cancer. $N$ Engl $J$ Med. 2017;377(20):1919-1929. doi:10.1056/NEJMoa1709937

17. Tawbi HA, Forsyth PA, Algazi A, et al. Combined nivolumab and ipilimumab in melanoma metastatic to the brain. $N$ Engl $\mathrm{J}$ Med. 2018;379(8):722-730. doi:10.1056/NEJMoa1805453

18. Margolin K, Ernstoff MS, Hamid O, et al. Ipilimumab in patients with melanoma and brain metastases: an open-label, Phase 2 trial. Lancet Oncol. 2012;13(5):459-465. doi:10.1016/S1470-2045(12) 70090-6

19. Goldberg SB, Gettinger SN, Mahajan A, et al. Pembrolizumab for patients with melanoma or non-small-cell lung cancer and untreated brain metastases: early analysis of a non-randomised, open-label, phase 2 trial. Lancet Oncol. 2016;17(7):976-983. doi:10.1016/ S1470-2045(16)30053-5

20. Minniti G, Anzellini D, Reverberi C, et al. Stereotactic radiosurgery combined with nivolumab or ipilimumab for patients with melanoma brain metastases: evaluation of brain control and toxicity. J Immunother Cancer. 2019;7(1):102. doi:10.1186/s40425-019-0588-y

21. Trapani S, Manicone M, Sikokis A, et al. Effectiveness and safety of "real" concurrent stereotactic radiotherapy and immunotherapy in metastatic solid tumors: a systematic review. Crit Rev Oncol Hematol. 2019;142(p):9-15. doi:10.1016/j.critrevonc.2019.07.006

22. Lehrer EJ, Peterson J, Brown PD, et al. Treatment of brain metastases with stereotactic radiosurgery and immune checkpoint inhibitors: an international meta-analysis of individual patient data. Radiother Oncol. 2019;130:104-112. doi:10.1016/j.radonc.2018.08.025

23. Chen L, Douglass J, Kleinberg L, et al. Redmond concurrent immune checkpoint inhibitors and stereotactic radiosurgery for brain metastases in non-small cell lung cancer, melanoma, and renal cell carcinoma. Int J Radiat Oncol Biol Phys. 2018;100(4):916-925. doi:10.1016/j.ijrobp.2017.11.041

24. Singh C, Qian JM, Yu JB, et al. Local tumor response and survival outcomes after combined stereotactic radiosurgery and immunotherapy in non-small cell lung cancer with brain metastases. J Neurosurg. 2020;132(2):512-517. doi:10.3171/2018.10.JNS181371 
25. Gauvain C, Vauléon E, Chouaid C, et al. Intracerebral efficacy and tolerance of nivolumab in non-small-cell lung cancer patients with brain metastases. Lung Cancer. 2018;116:62-66. doi:10.1016/j. lungcan.2017.12.008

26. Bidoli P, Chiari R, Catino A, et al. Efficacy and safety data from patients with advanced squamous NSCLC and brain metastases participating in the nivolumab Expanded Access Programme (EAP) in Italy. Ann Oncol. 2016;27:1228P. doi:10.1093/annonc/mdw383.28

27. Karivedu V, Jandarov JR, Wise-Draper TM. Brain metastases treated with immune checkpoint inhibitors: a single center experience. J Clin Oncol. 2018;36(15_suppl):e14012. doi:10.1200/JCO.2018.36. 15_suppl.e14012

28. Di Giacomo AM, Ascierto PA, Queirolo P, et al. Three-year follow-up of advanced melanoma patients who received ipilimumab plus fotemustine in the Italian Network for Tumor Biotherapy (NIBIT)-M1 phase II study. Ann Oncol. 2015;26(4):798-803. doi:10.1093/annonc/mdu577

29. Long GV, Atkinson V, Lo S, et al. Combination nivolumab and ipilimumab or nivolumab alone in melanoma brain metastases: a multicentre randomised phase 2 study. Lancet Oncol. 2018;19 (5):672-681. doi:10.1016/S1470-2045(18)30139-6

30. Gandhi L, Rodríguez-Abreu D, Gadgeel S, et al. Pembrolizumab plus chemotherapy in metastatic non-small-cell lung cancer. $N$ Engl J Med. 2018;378(22):2078-2092. doi:10.1056/NEJMoa1801005

31. Paz-Ares L, Luft A, Vicente D, et al. Pembrolizumab plus chemotherapy for squamous non-small-cell lung cancer. $N$ Engl J Med. 2018;379(21):2040-2051. doi:10.1056/NEJMoa1810865

32. West H, McCleod M, Hussein M, et al. Atezolizumab in combination with carboplatin plus nab-paclitaxel chemotherapy compared with chemotherapy alone as first-line treatment for metastatic non-squamous non-small-cell lung cancer (IMpower130): a multicentre, randomised, open-label, Phase 3 trial. Lancet Oncol. 2019;20(7):924-937. doi:10.1016/S1470-2045(19)30167-6
33. Hodge JW, Guha C, Neefjes J, Gulley JL. Synergizing radiation therapy and immunotherapy for curing incurable cancers. Opportunities and challenges. Oncology. 2008;22:1064-1070.

34. Tang C, Wang X, Soh $\mathrm{H}$, et al. Combining radiation and immunotherapy: a new systemic therapy for solid tumors? Cancer Immunol Res. 2014;2(9):831-838. doi:10.1158/2326-6066.CIR-14-0069

35. Dovedi SJ, Adlard AL, Lipowska-Bhalla G, et al. Acquired resistance to fractionated radiotherapy can be overcome by concurrent PD-L1 blockade. Cancer Res. 2014;74(19):5458-5468. doi:10.1158/00085472.CAN-14-1258

36. Diao K, Bian SX, Routman DM, et al. Combination ipilimumab and radiosurgery for brain metastases: tumor, edema, and adverse radiation effects. J Neurosurg. 2018;129:1379-1406. doi:10.3171/2017.7. JNS171286

37. Ahmed KA, Kim S, Arrington J, et al. Outcomes targeting the PD-1/ PD-L1 axis in conjunction with stereotactic radiation for patients with non-small cell lung cancer brain metastases. $J$ Neurooncol. 2017;133(2):331-338. doi:10.1007/s11060-017-2437-5

38. Demaria S, Formenti SC. Radiation as an immunological adjuvant: current evidence on dose and fractionation. Front Oncol. 2012;2:153. doi:10.3389/fonc. 2012.00153

39. Hendriks LEL, Henon C, Auclin E, et al. Outcome of patients with non-small cell lung cancer and brain metastases treated with checkpoint inhibitors. J Thorac Oncol. 2019;14(7):1244-1254. doi:10.10 16/j.jtho.2019.02.009

40. Goldberg SB, Schalper KA, Gettinger SN, et al. Pembrolizumab for management of patients with NSCLC and brain metastases: long-term results and biomarker analysis from a non-randomised, open-label, phase 2 trial. Lancet Oncol. 2020;21(5):655-663. doi:10.1016/S1470-2045(20)30111-X

\section{Publish your work in this journal}

OncoTargets and Therapy is an international, peer-reviewed, open access journal focusing on the pathological basis of all cancers, potential targets for therapy and treatment protocols employed to improve the management of cancer patients. The journal also focuses on the impact of management programs and new therapeutic agents and protocols on patient perspectives such as quality of life, adherence and satisfaction. The manuscript management system is completely online and includes a very quick and fair peer-review system, which is all easy to use. Visit http://www.dovepress.com/ testimonials.php to read real quotes from published authors 